\title{
Temperature dependence of recombination radiation in semiconductor nanostructures with quantum dots containing impurity complexes
}

\author{
V. D. Krevchik ${ }^{1}$, A. V. Razumov ${ }^{1}$, M. B. Semenov ${ }^{1}$, I. M. Moyko ${ }^{1}$, A. V. Shorokhov ${ }^{1,2}$ \\ ${ }^{1}$ Penza State University, Krasnaya, 40, Penza, 440026, Russia \\ ${ }^{2}$ University of Jyväskylä, PO Box 35, Jyväskylä, FI-40014, Finland \\ physics@pnzgu.ru, alex.shorokhov@gmail.com
}

PACS 73.40.Gk, 03.65.Xp

DOI 10.17586/2220-8054-2021-12-6-680-689

\begin{abstract}
Temperature dependence of the spectral intensity of recombination radiation in a quasi-zero-dimensional structure, containing impurity complexes " $A^{+}+e "$ (a hole localized on a neutral acceptor, interacting with an electron localized in the ground state of a quantum dot), has been investigated in an external electric field in the presence of tunneling decay of a quasistationary $A^{+}$-state. Probability of dissipative tunneling of a hole has been calculated in the one-instanton approximation, and the influence of tunneling decay and of an external electric field on the $A^{+}$-state binding energy and on the spectra of recombination radiation, associated with the optical transition of an electron from the ground state of a quantum dot to the $A^{+}$-state of the impurity center, has been investigated in the adiabatic approximation. "Dips" in the temperature dependence of the SIRR have been revealed, which are associated with the presence of resonant tunneling at certain values of temperature and strength of the external electric field, for which the double-well oscillatory potential becomes symmetric.
\end{abstract}

Keywords: spectral intensity of recombination radiation, quasi-zero-dimensional structure, impurity complexes, quantum dots.

Received: 15 November 2021

Revised: 19 November 2021

\section{Introduction}

The relevance of studies of the spectral intensity of recombination radiation (SIRR) temperature dependence in quasi-zero-dimensional structures is determined by the fact that, first, electro-optical systems based on quantum dots (QD)s have significantly better parameters compared to similar devices based on quantum wells. Second, the temperature quenching of luminescence is usually associated with the presence of a nonradiative channel caused by a defect located in a QD [1,2] or at the barrier boundary. In this work, we would like to draw attention to the possible existence of one more channel of temperature quenching of luminescence associated with tunneling processes, in particular, with the process of dissipative tunneling of a hole localized at the $A^{+}$-center into the matrix, surrounding the QD. As will be shown below, the temperature "spreading" of the wave function for the quasi-stationary $A^{+}$-state under tunnel decay conditions is accompanied by an increase in the energy of the radiative transition of an electron and a corresponding decrease in the overlap integral of the wave functions of a hole and an electron, which leads to temperature quenching of the recombination radiation. The aim of this work is to study theoretically the temperature effect, associated with the electron - phonon interaction, on the binding energy of a hole in the " $A^{+}+e^{\text {"-impurity }}$ complex in a spherically symmetric QD, as well as on the spectral intensity of the recombination radiation of a quasizero-dimensional structure in the presence of a dissipative tunneling.

\section{Binding energy of a quasi-stationary $A^{+}$-state in a quantum dot in the presence of dissipative tunneling in} an external electric field

Let us consider the problem of quasi-stationary states of a hole in an impurity complex $A^{+}+e$ in a semiconductor spherically symmetric QD. The interaction of an electron in the QD ground state with a hole, localized at the $A^{0}$-center, will be considered in the framework of the adiabatic approximation [3]. In this case, the electron potential $V_{n, l, m}(\vec{r})$ acting on the hole can be considered averaged over the electron motion:

$$
V_{n l m}(\vec{r})=-\frac{e^{2}}{4 \pi \varepsilon_{0} \varepsilon} \int_{0}^{R_{0}} \frac{\left|\Psi_{n l m}\left(\vec{r}_{e}\right)\right|^{2}}{\left|\vec{r}-\vec{r}_{e}\right|} d \vec{r}_{e},
$$

where $e$ is the the electron charge; $\varepsilon$ is the dielectric permittivity of QD material; $\varepsilon_{0}$ is the electrical constant; $m=$ $0, \pm 1, \pm 2, \ldots$ is the magnetic quantum number; $l=0,1,2 \ldots$ is the orbital quantum number, $\Psi_{n l m}\left(\vec{r}_{e}\right)$ is the wave 
function of an electron in a QD determined by the expression (2):

$$
\Psi_{n l m}\left(\vec{r}_{e}\right)=Y_{l m}\left(\theta_{e}, \varphi_{e}\right) \frac{J_{l+\frac{3}{2}}\left(K_{n l} r_{e}\right)}{\sqrt{2 \pi} R_{0} \sqrt{r} J_{l+\frac{3}{2}}\left(K_{n l} R_{0}\right)},
$$

where $K_{n l}$ is defined by an expression of the following form:

$$
K_{n l}=\sqrt{\frac{\tilde{X}_{n, l}^{2}}{R_{0}^{* 2}}+\frac{k T}{E_{h}} \ln \left[4 \sinh \left(\frac{\Omega}{v_{L A} \sqrt{k T}}\right) \sinh \left(\frac{2 \Omega}{v_{T A} \sqrt{k T}}\right)\right]} .
$$

Here $\nu_{L A}, \nu_{T A}$ are the velocities of longitudinal and transverse phonons; $\Omega=\frac{\pi \hbar \Theta G}{a} \sqrt{\frac{2}{\rho V}}, G$ is the overlap integral, $\Theta$ is the deformation potential, $a$ is the lattice constant, $\rho$ is the QD material density, $V$ is the QD volume, $E_{h}$ is the Bohr hole energy, $\tilde{X}_{n, l}$ is the half-integer root of the Bessel function $l+1 / 2$.

Let us consider the case corresponding to the $p$-state of an electron, in this case $l=1, m=0$, then expression (1) can be represented in the form

$$
\begin{aligned}
V_{n 10}\left(\vec{r}_{h}\right) & = \\
& -\frac{e^{2}}{4 \pi \varepsilon_{0} \varepsilon} \int_{0}^{\pi} \sin \theta_{e} d \theta_{e} \int_{0}^{2 \pi} d \varphi_{e}\left(\int_{0}^{r_{h}} \frac{\left|\Psi_{n 10}\left(r_{e}, \theta_{e}, \varphi_{e}\right)\right|^{2} r_{e}^{2} d r_{e}}{\sqrt{r_{h}^{2}+r_{e}^{2}-2 r_{h} r_{e} \cos \theta_{e}}}+\int_{r_{h}}^{R_{0}} \frac{\left|\Psi_{n 10}\left(r_{e}, \theta_{e}, \varphi_{e}\right)\right|^{2} r_{e}^{2} d r_{e}}{\sqrt{r_{h}^{2}+r_{e}^{2}-2 r_{h} r_{e} \cos \theta_{e}}}\right) .
\end{aligned}
$$

Or, taking into account the explicit expression for the wave function:

$$
\begin{array}{r}
V_{n 10}\left(\vec{r}_{h}\right)=-\frac{3 e^{2}}{32 \pi^{3} \varepsilon_{0} \varepsilon R_{0}^{2} J_{\frac{5}{2}}^{2}\left(K_{n 1} R_{0}\right)} \int_{0}^{\pi} \sin \theta_{e} \cos ^{2} \theta_{e} d \theta_{e} \int_{0}^{2 \pi} d \varphi_{e}\left(\int_{0}^{r_{h}} \frac{J_{\frac{5}{2}}^{2}\left(K_{n 1} r_{e}\right) r_{e} d r_{e}}{\sqrt{r_{h}^{2}+r_{e}^{2}-2 r_{h} r_{e} \cos \theta_{e}}}\right. \\
\left.+\int_{r_{h}}^{R_{0}} \frac{J_{\frac{5}{2}}^{2}\left(K_{n l} r_{e}\right) r_{e} d r_{e}}{\sqrt{r_{h}^{2}+r_{e}^{2}-2 r_{h} r_{e} \cos \theta_{e}}}\right)
\end{array}
$$

After integration over the angular variables $\varphi_{e}, \theta_{e}$ and over the radial coordinate of the electron $r_{e}$, we obtain:

$$
\begin{aligned}
& V_{n 10}\left(r_{h}\right)=\frac{e^{2}}{2^{7} \pi^{3} \varepsilon_{0} \varepsilon R_{0}^{* 6} K_{n 1}^{* 5} r_{h}^{* 4} J_{\frac{5}{2}}^{2}\left(K_{n 1}^{*} r_{h}^{*}\right)}\left[3 R_{0}^{* 4}+6 K_{n 1}^{* 2} R_{0}^{* 4} r_{h}^{* 2}+9 r_{h}^{* 4}+6 K_{n 1}^{* 2} R_{0}^{* 2} r_{h}^{* 4}\right. \\
& -4 K_{n 1}^{* 4} R_{0}^{* 4} r_{h}^{* 4}-9 r_{h}^{* 4} \cos \left(2 K_{n 1}^{*} R_{0}^{*}\right)+12 K_{n 1}^{* 2} R_{0}^{* 2} r_{h}^{* 4} \cos \left(2 K_{n 1}^{*} R_{0}^{*}\right) \\
& -3 R_{0}^{* 4} \cos \left(2 K_{n 1}^{*} R_{0}^{*}\right)+4 K_{n 1}^{* 4} R_{0}^{* 4} r_{h}^{* 4}\left(\mathrm{Ci}\left(2 K_{n 1}^{*} R_{0}^{*}\right)-\mathrm{Ci}\left(2 K_{n 1}^{*} r_{h}^{*}\right)\right) \\
& \left.-4 K_{n 1}^{* 4} R_{0}^{* 4} r_{h}^{* 4} \ln \left(\frac{R_{0}^{*}}{r_{h}^{*}}\right)-\left(9 r_{h}^{* 3}+3 R_{0}^{* 3}-K_{n 1}^{* 2} R_{0}^{* 3} r_{h}^{* 2}\right) 2 K_{n 1}^{*} R_{0}^{*} r_{h}^{*} \sin \left(2 K_{n 1}^{*} R_{0}^{*}\right)\right] .
\end{aligned}
$$

Here, $r_{h}^{*}=r_{h} / a_{h}, R_{0}^{*}=R_{0} / a_{h}$,

$$
K_{n}^{*}=\sqrt{\frac{X_{n, l}^{2}}{R_{0}^{* 2}}+\frac{k T}{E_{h} R_{0}^{* 2}} \ln \left[4 \sinh \left(\frac{\Omega}{v_{L A} \sqrt{k T}}\right) \sinh \left(\frac{2 \Omega}{v_{T A} \sqrt{k T}}\right)\right]}
$$

$\mathrm{Ci}(x)$ is the cosine integral.

In the case under consideration, $p$-state with $m=0$, the potential energy minimum is shifted relative to the QD center. Position of the minimum $r_{h \text { min }}$ is determined from the solution of the transcendental equation, which is obtained by equating the first derivative of expression (6) to zero:

$$
\frac{d V_{n, 1,0}\left(r_{h}\right)}{d r_{h}}=0
$$


Expanding expression (6) in a Taylor series near $r_{h \text { min }}$ and limiting ourselves to the quadratic term in the radial coordinate of the hole $r_{h}$, we obtain:

$$
\begin{gathered}
V_{n 10}\left(r_{h}\right)=\frac{e^{2} 2^{-7} \pi^{-3}}{\varepsilon_{0} \varepsilon R_{0}^{* 6} K_{n 1}^{* 5} r_{h \min }^{* 6} J_{5 / 2}^{2}\left(K_{n 1}^{*} R_{0}^{*}\right)}\left[r_{h \min }^{* 2}\left(2 K_{n 1}^{* 2} R_{0}^{* 2} r_{h \min }^{* 2}\left(3 R_{0}^{* 2}+3 r_{h \min }^{* 2}-2 K_{n 1}^{* 2} R_{0}^{* 2} r_{h \min }^{* 2}\right)\right)+\right. \\
+4 K_{n 1}^{* 4} R_{0}^{* 4} r_{h \min }^{* 4}\left(\mathrm{Ci}\left(2 K_{n 1}^{*} R_{0}^{*}\right)-\mathrm{Ci}\left(2 K_{n 1}^{*} r_{h \min }^{*}\right)-\ln \left(\frac{R_{0}^{*}}{r_{h \min }^{*}}\right)\right)- \\
-2 K_{n 1}^{*} r_{h \min }^{*} R_{0}^{*}\left(K_{n 1}^{* 2} R_{0}^{* 3} r_{h \min }^{* 2} \sin \left(2 K_{n 1}^{*} r_{h \min }^{*}\right)-9 r_{h \min }^{* 3} \sin \left(2 K_{n 1}^{*} R_{0}^{*}\right)-3 R_{0}^{* 3} \sin \left(2 K_{n 1}^{*} r_{h \min }^{*}\right)\right)+ \\
+\left(r_{h}^{*}-r_{h \min }^{*}\right) 2 R_{0}^{* 4} r_{h \min }^{*}\left(-6-6 K_{n 1}^{* 2} r_{h \min }^{* 2}+2 K_{n 1}^{* 4} r_{h \min }^{* 4}+6\left(1-K_{n 1}^{* 2} r_{h \min }^{* 2}\right) \cos \left(2 K_{n 1}^{*} r_{h \min }^{*}\right)+\right. \\
\left.+K_{n 1}^{*} r_{h \min }^{*}\left(K_{n 1}^{* 2} r_{h \min }^{* 2}-12\right) \sin \left(2 K_{n 1}^{*} r_{h \min }^{*}\right)\right)- \\
-\left(r_{h}^{*}-r_{h \min }^{*}\right)^{2} 2 R_{0}^{* 4}\left(-15-9 K_{n 1}^{* 2} r_{h \min }^{* 2}+K_{n 1}^{* 4} r_{h \min }^{* 4}+\right. \\
\left.\left.+\left(15-21 K_{n 1}^{* 2} r_{h \min }^{* 2}+K_{n 1}^{* 4} r_{h \min }^{* 4}\right) \cos \left(2 K_{n 1}^{*} r_{h \min }^{*}\right)+\left(30 K_{n 1}^{*} r_{h \min }^{*}-7 K_{n 1}^{* 3} r_{h \min }^{* 3}\right) \sin \left(2 K_{n 1}^{*} r_{h \min }^{*}\right)\right)\right]
\end{gathered}
$$

where $r_{h \min }^{*}=r_{h \min } / a_{h}$.

Within the framework of the adiabatic approximation for the $p$-state of an electron, we obtain:

$$
V_{n 10}\left(r_{h}^{*}\right)=-\frac{e^{2}}{\varepsilon R_{0}^{*}} \beta_{n}-\frac{m_{h} \omega_{n}^{2}\left(r_{h}^{*}-r_{T}^{*}\right)^{2}}{2}
$$

where values $\beta_{n}, \omega_{n}$ and $r_{T}^{*}$ are defined as follows:

$$
\begin{aligned}
& \beta_{n}=\frac{2^{-7} \pi^{-3}}{R_{0}^{* 5} K_{n 1}^{* 5} r_{h \min }^{* 6} J_{5 / 2}^{2}\left(K_{n 1}^{*} R_{0}^{*}\right)}\left[r_{h \min }^{* 2}\left(2 K_{n 1}^{* 2} R_{0}^{* 2} r_{h \min }^{* 2}\left(3 R_{0}^{* 2}+3 r_{h \min }^{* 2}-2 K_{n 1}^{* 2} R_{0}^{* 2} r_{h \min }^{* 2}\right)\right)+\right. \\
& +3 R_{0}^{* 4}+9 r_{h \min }^{* 4}+3 r_{h \min }^{* 4} \cos \left(2 K_{n 1}^{*} R_{0}^{*}\right)\left(6 K_{n 1}^{* 2} R_{0}^{* 2}-3\right)-3 R_{0}^{* 4} \cos \left(2 K_{n 1}^{*} r_{h \min }^{*}\right)+ \\
& +4 K_{n 1}^{* 4} R_{0}^{* 4} r_{h \min }^{* 4}\left(\mathrm{Ci}\left(2 K_{n 1}^{*} R_{0}^{*}\right)-\mathrm{Ci}\left(2 K_{n 1}^{*} r_{h \min }^{*}\right)-\ln \left(\frac{R_{0}^{*}}{r_{h \min }^{*}}\right)\right)- \\
& -2 K_{n 1}^{*} r_{h \min }^{*} R_{0}^{*}\left(K_{n 1}^{* 2} R_{0}^{* 3} r_{h \min }^{* 2} \sin \left(2 K_{n 1}^{*} r_{h \min }^{*}\right)-9 r_{h \min }^{* 3} \sin \left(2 K_{n 1}^{*} R_{0}^{*}\right)-3 R_{0}^{* 3} \sin \left(2 K_{n 1}^{*} r_{h \min }^{*}\right)\right)+ \\
& +2 R_{0}^{* 4} r_{h \min }^{* 2}\left(-6-6 K_{n 1}^{* 2} r_{h \min }^{* 2}+2 K_{n 1}^{* 4} r_{h \text { min }}^{* 4}+6\left(1-K_{n 1}^{* 2} r_{h \min }^{* 2}\right) \cos \left(2 K_{n 1}^{*} r_{h \min }^{*}\right)+\right. \\
& \left.+K_{n 1}^{*} r_{h \min }^{*}\left(K_{n 1}^{* 2} r_{h \min }^{* 2}-12\right) \sin \left(2 K_{n 1}^{*} r_{h \min }^{*}\right)\right)^{2} \times\left(15+9 K_{n 1}^{* 2} r_{h \min }^{* 2}-K_{n 1}^{* 4} r_{h \min }^{* 4}-\right. \\
& \left.\left.-\cos \left(2 K_{n 1}^{*} r_{h \min }^{*}\right)\left(15-21 K_{n 1}^{* 2} r_{h \min }^{* 2}+K_{n 1}^{* 4} r_{h \min }^{* 4}\right)-\left(30 K_{n 1}^{*} r_{h \min }^{*}-7 K_{n 1}^{* 3} r_{h \min }^{* 3}\right) \sin \left(2 K_{n 1}^{*} r_{h \min }^{*}\right)\right)^{-1}\right] \\
& \hbar \omega_{n}=\left[\frac { \hbar ^ { 2 } e ^ { 2 } 2 ^ { - 5 } \pi ^ { - 3 } } { m _ { h } ^ { * } \varepsilon _ { 0 } \varepsilon R _ { 0 } ^ { * 2 } K _ { n 1 } ^ { * 5 } r _ { h \operatorname { m i n } } ^ { * 6 } J _ { 5 / 2 } ^ { 2 } ( K _ { n 1 } R _ { 0 } ^ { * } ) } \left(15+9 K_{n 1}^{* 2} r_{h \min }^{* 2}-K_{n 1}^{* 4} r_{h \min }^{* 4}-\right.\right. \\
& \left.\left.-\cos \left(2 K_{n 1}^{*} r_{h \min }^{*}\right)\left(15-21 K_{n 1}^{* 2} r_{h \min }^{* 2}+K_{n 1}^{* 4} r_{h \min }^{* 4}\right)-\left(30 K_{n 1}^{*} r_{h \min }^{*}-7 K_{n 1}^{* 3} r_{h \min }^{* 3}\right) \sin \left(2 K_{n 1}^{*} r_{h \min }^{*}\right)\right)\right]^{1 / 2}
\end{aligned}
$$

and

$$
\begin{aligned}
& r_{T}^{*}=-r_{h \min }^{*}-\frac{r_{h \min }^{*}}{2}\left(-6-6 K_{n 1}^{* 2} r_{h \min }^{* 2}+2 K_{n 1}^{* 4} r_{h \min }^{* 4}+6\left(1-K_{n 1}^{* 2} r_{h \min }^{* 2}\right) \cos \left(2 K_{n 1}^{*} r_{h \min }^{*}\right)+\right. \\
& \left.\quad+K_{n 1}^{*} r_{h \min }^{*}\left(K_{n 1}^{* 2} r_{h \min }^{* 2}-12\right) \sin \left(2 K_{n 1}^{*} r_{h \min }^{*}\right)\right) \times\left[-15-9 K_{n 1}^{* 2} r_{h \min }^{* 2}+K_{n 1}^{* 4} r_{h \min }^{* 4}+\right. \\
& \left.+\left(15-21 K_{n 1}^{* 2} r_{h \min }^{* 2}+K_{n 1}^{* 4} r_{h \min }^{* 4}\right) \cos \left(2 K_{n 1}^{*} r_{h \min }^{*}\right)+\left(30 K_{n 1}^{*} r_{h \min }^{*}-7 K_{n 1}^{* 3} r_{h \min }^{* 3}\right) \sin \left(2 K_{n 1}^{*} r_{h \min }^{*}\right)\right]^{-1}
\end{aligned}
$$


Since the confining potential of a QD, generally speaking, should have a finite depth, then in our model of the hole potential of confinement (9), the amplitude of the potential $U_{0}$ is an empirical parameter and satisfies the relation

$$
U_{0}=-e^{2} \beta_{n} / 4 \pi \varepsilon_{0} \varepsilon R_{0}+m_{h}^{*} \omega_{n}^{2} R_{0}^{2} / 2=m_{h}^{*} \omega_{0}^{2} R_{0}^{2} / 2,
$$

whence $\omega_{0}=\sqrt{\omega_{n}^{2}-e^{2} \beta_{n} / 2 \pi \varepsilon_{0} \varepsilon m_{h} R_{0}^{3}}$ is the characteristic frequency of the hole confining potential of a QD within the adiabatic approximation, and in this case $U_{0} /\left(\hbar \omega_{0}\right) \gg 1$.

Usage of the adiabatic approximation makes it possible to take into account the effect of an external electric field on the bound state of the hole. Let the electric field strength vector $\vec{E}_{0}$ be directed along the $x$ coordinate axis, then the energy levels of the oscillatory potential (9) will have the form:

$$
\begin{aligned}
E_{n_{1} n_{2} n_{3}}^{n, 0,0}(T)=-\frac{e^{2}}{\varepsilon R_{0}} \beta_{h}-\frac{|e|^{2} E_{0}^{2}}{2 m_{h} \omega_{n}^{2}}+\hbar \omega_{n}\left(n_{1}+n_{2}\right. & \left.+n_{3}+\frac{3}{2}\right)+ \\
& +k T \ln \left[4 \sinh \left(\frac{\Omega}{v_{L A} \sqrt{k T}}\right) \sinh \left(\frac{2 \Omega}{v_{T A} \sqrt{k T}}\right)\right],
\end{aligned}
$$

and the corresponding one-particle wave functions are written as:

$$
\Psi_{n_{1} n_{2} n_{3}}^{n}(x, y, z)=C_{n} \exp \left(-\frac{\left(x-x_{0}\right)^{2}+y^{2}+z^{2}}{2 a_{n}^{2}}\right) H_{n_{1}}\left(\frac{x-x_{0}}{a_{n}}\right) H_{n_{2}}\left(\frac{y}{a_{n}}\right) H_{n_{3}}\left(\frac{z}{a_{n}}\right) .
$$

Here, $C_{n}=\left[2^{n 1+n 2+n 3} n_{1} ! n_{2} ! n_{3} ! \pi^{3 / 2} a_{n}^{3}\right]^{-1 / 2} ; a_{n}=\sqrt{\hbar /\left(m_{h}^{*} \omega_{n}\right)} ; x_{0}=|e| E_{0} /\left(m_{h}^{*} \omega_{n}^{2}\right) ; H_{n}(x)$ are the Hermite polynomials; $n_{1}, n_{2}, n_{3}$ are the quantum numbers corresponding to energy levels of a harmonic oscillator. We will assume that the process of decay of the quasi-stationary level of the $A^{+}$-center is due to dissipative tunneling. It should be noted that in the one-instanton approximation, the decay probability $\Gamma_{0}$ (dissipative tunneling), under conditions of an external electric field, can be represented in the form $\Gamma_{0}=B \exp (-S)$, where the expressions for $S$ and $B$ were obtained in our previous papers (in the Bohr units) [4,5]:

$$
\begin{aligned}
& S=\frac{1}{2}\left(\frac{b_{0}^{\prime}+x_{0}^{*}}{a_{0}^{\prime}+x_{0}^{*}}+1\right)\left(3-\frac{b_{0}^{\prime}+x_{0}^{*}}{a_{0}^{\prime}+x_{0}^{*}}\right) \tau_{0}^{*}-\frac{1}{2 \beta^{*}}\left(\frac{b_{0}^{\prime}+x_{0}^{*}}{a_{0}^{\prime}+x_{0}^{*}}+1\right)^{2} \tau_{0}^{* 2}-\frac{1}{2 \gamma^{*}}\left(\frac{b_{0}^{\prime}+x_{0}^{*}}{a_{0}^{\prime}+x_{0}^{*}}+1\right)^{2} \times \\
& \times\left(\frac{\left(1-x_{2}^{*}\right)}{\sqrt{x_{1}^{*}}}\left[\operatorname{coth}\left(\beta^{*} \sqrt{x_{1}^{*}}\right)-\frac{\cosh \left(\left(\beta^{*}-\tau_{0}^{*}\right) \sqrt{x_{1}^{*}}\right)-\cosh \left(\beta^{*} \sqrt{x_{1}^{*}}\right)}{\sinh \left(\beta^{*} \sqrt{x_{1}^{*}}\right)}+\cosh \left(\left(\beta^{*}-\tau_{0}^{*}\right) \sqrt{x_{1}^{*}}\right)\right]-\right. \\
& \left.-\frac{\left(1-x_{1}^{*}\right)}{\sqrt{x_{2}^{*}}}\left[\operatorname{coth}\left(\beta^{*} \sqrt{x_{2}^{*}}\right)-\frac{\cosh \left(\left(\beta^{*}-\tau_{0}^{*}\right) \sqrt{x_{2}^{*}}\right)-\cosh \left(\beta^{*} \sqrt{x_{2}^{*}}\right)}{\sinh \left(\beta^{*} \sqrt{x_{2}^{*}}\right)}+\cosh \left(\left(\beta^{*}-\tau_{0}^{*}\right) \sqrt{x_{2}^{*}}\right)\right]\right) \\
& B=\frac{2 E_{d} \sqrt{U_{0}^{*}}}{\hbar \sqrt{\pi}}\left(\frac{b_{0}^{\prime}+x_{0}^{*}}{a_{0}^{\prime}+x_{0}^{*}}+1\right) \sqrt{\varepsilon_{T}^{*}} \times\left\{A^{*}\left[\beta_{1}^{*} \cosh \left(\frac{\beta_{1}^{*}}{2}\right)-1\right]+D^{*}\left[\beta_{2}^{*} \cosh \left(\frac{\beta_{2}^{*}}{2}\right)-1\right]+\right. \\
& \left.+A^{*}\left(1-\frac{\beta_{1}^{*}}{2} \frac{\left(\frac{\beta_{1}^{*}}{2}-\tau_{01}^{*}\right)}{\sinh \left(\frac{\beta_{1}^{*}}{2}\right)}\right)+D^{*}\left(\frac{\beta_{2}^{*}}{2} \frac{\cosh \left(\frac{\beta_{2}^{*}}{2}-\tau_{02}{ }^{*}\right)}{\sinh \left(\frac{\beta_{2}^{*}}{2}\right)}-1\right)\right\} \times \\
& \left(A^{*}\left[\frac{\beta_{1}^{*}}{2} \frac{\cosh \left(\frac{\beta_{1}^{*}}{2}-\tau_{01}{ }^{*}\right)}{\sinh \left(\frac{\beta_{1}^{*}}{2}\right)}-1\right]+D^{*}\left[\frac{\beta_{2}^{*}}{2} \frac{\cosh \left(\frac{\beta_{2}^{*}}{2}-\tau_{02}{ }^{*}\right)}{\sinh \left(\frac{\beta_{2}^{*}}{2}\right)}-1\right]\right)^{-1 / 2}
\end{aligned}
$$

where

$$
\begin{gathered}
x_{1,2}^{*}=\frac{1}{2}\left[\frac{\varepsilon_{L}^{* 2} a^{* 2}}{4 U_{0}^{*}}+1+\frac{\varepsilon_{c}^{4} a^{* 2}}{4 \varepsilon_{L}^{* 2} U_{0}^{*}} \mp \sqrt{\left.\left(\frac{\varepsilon_{L}^{* 2} a^{* 2}}{4 U_{0}^{*}}+1+\frac{\varepsilon_{c}^{4} a^{* 2}}{4 \varepsilon_{L}^{* 2} U_{0}^{*}}\right)^{2}-\frac{\varepsilon_{L}^{* 2} a^{* 2}}{U_{0}^{*}}\right],}\right. \\
\gamma^{*}=\sqrt{\left(\varepsilon_{L}^{* 2} a^{* 2} /\left(4 U_{0}^{*}\right)+1+\varepsilon_{c}^{* 4} a^{* 2} /\left(4 \varepsilon_{L}^{* 2} U_{0}^{*}\right)\right)^{2}-\varepsilon_{L}^{* 2} a^{* 2} / U_{0}^{*},} \\
\tau_{0}^{*}=\operatorname{arsinh}\left[\left(1-\frac{b_{0}^{\prime}+x_{0}^{*}}{a_{0}^{\prime}+x_{0}^{*}}\right) \sinh \left(\beta^{*}\right) /\left(1+\frac{b_{0}^{\prime}+x_{0}^{*}}{a_{0}^{\prime}+x_{0}^{*}}\right)\right]+\beta_{,}^{*}, \\
\varepsilon_{T}^{*}=k T / E_{h}, \quad \varepsilon_{L}^{*}=\hbar \omega_{L} / E_{h}, \quad \varepsilon_{c}^{*}=\hbar \sqrt{c} / E_{d}, \quad \beta^{*}=\sqrt{U_{0}^{*}} / a^{*} \varepsilon_{T}^{*}, \quad b_{0}^{\prime}=b_{0} / a_{h}, \quad a_{0}^{\prime}=a_{0} / a_{h}, \quad x_{0}^{*}=x_{0} / a_{h} ;
\end{gathered}
$$


$E_{h}$ and $a_{h}$ are the Bohr energy and the hole radius correspondingly;

$$
\begin{gathered}
A^{*}=\left(2 \varepsilon_{L}^{* 2} a^{* 2}-x_{1}^{*}\right) /\left(\left(x_{1}^{*}-x_{2}^{*}\right) x_{1}^{*}\right), \quad D^{*}=\left(2 \varepsilon_{L}^{* 2} a^{* 2}-x_{2}^{*}\right) /\left(\left(x_{1}^{*}-x_{2}^{*}\right) x_{2}^{*}\right), \\
\beta_{1}^{*}=\sqrt{2 U_{0}^{*} x_{1}^{*}} /\left(a^{*} \varepsilon_{T}^{*}\right), \quad \beta_{2}^{*}=\sqrt{2 U_{0}^{*} x_{2}^{*}} /\left(a^{*} \varepsilon_{T}^{*}\right), \quad \tau_{01}^{*}=\tau_{0}^{*} \sqrt{x_{1}^{*}} / \sqrt{2}, \quad \tau_{02}^{*}=\tau_{0}^{*} \sqrt{x_{2}^{*}} / \sqrt{2} .
\end{gathered}
$$

Using the zero-range potential procedure (see, for example, [3]), in combination with the one-instanton approximation [5], we obtain an equation that determines the dependence of the hole energy in the complex $A^{+}+e$ on temperature $T$, QD parameters, and dissipative tunneling.

The short-range potential of an impurity is simulated by a potential of zero radius with a power $\gamma=2 \pi \hbar^{2} /\left(\alpha m_{h}^{*}\right)$, which has the form:

$$
V_{\delta}\left(x, y, z ; x_{a}, y_{a}, z_{a}\right)=\gamma \delta\left(x-x_{a}\right) \delta\left(y-y_{a}\right) \delta\left(z-z_{a}\right)\left[1+\left(r-r_{a}\right) \frac{\partial}{\partial r}\right],
$$

where $\alpha$ is determined by the binding energy $E_{i}$ of the same $A^{+}$-center in a bulk semiconductor.

In the effective mass approximation, the wave function $\Psi_{\lambda h}\left(x, y, z ; x_{a}, y_{a}, z_{a}\right)$ of an electron localized at a shortrange potential satisfies the Schrödinger equation:

$$
\left(E_{\lambda h}-H\right) \Psi_{\lambda h}\left(x, y, z ; x_{a}, y_{a}, z_{a}\right)=V_{\delta}\left(x, y, z ; x_{a}, y_{a}, z_{a}\right) \Psi_{\lambda h}\left(x, y, z ; x_{a}, y_{a}, z_{a}\right),
$$

where $E_{h}^{Q D}=-\hbar^{2} \lambda^{2} /\left(2 m_{h}^{*}\right)$ - the eigenvalues of the Hamilton operator $H^{\delta}=H+V_{\delta}\left(x, y, z ; x_{a}, y_{a}, z_{a}\right) ; H=$ $-\hbar^{2} /\left(2 m_{h}^{*}\right) \nabla^{2}+m_{h}^{*} \omega_{0}^{2}\left(x^{2}+y^{2}+z^{2}\right) / 2-|e| E_{0} x$.

To determine the binding energy of a hole in a complex $A^{+}+e$ in the adiabatic approximation, it is necessary to construct a one-particle Green's function $G\left(x, y, z ; x_{a}, y_{a}, z_{a} ; E_{\lambda n}\right)$ to the Schrödinger equation with a Hamiltonian, containing potential (17):

$$
G\left(x, y, z ; x_{a}, y_{a}, z_{a} ; E_{\lambda h}\right)=-\sum_{n 1, n 2, n 3} \frac{\Psi_{n_{1}, n_{2}, n_{3}}^{n *}\left(x_{a}, y_{a}, z_{a}\right) \Psi_{n_{1}, n_{2}, n_{3}}^{n}(x, y, z)}{-E_{h}^{Q D}+i \hbar \Gamma_{0}+E_{n_{1}, n_{2}, n_{3}}^{n, 0}(T)} .
$$

The Lippmann-Schwinger equation for a $A^{+}$-state in a QD with a parabolic confinement potential can be written as:

$$
\begin{aligned}
\Psi_{h}\left(x, y, z ; x_{a}, y_{a}, z_{a}\right)= & \int_{-\infty}^{+\infty} \int_{-\infty}^{+\infty} \int_{-\infty}^{+\infty} d x_{1} d y_{1} d z_{1} G\left(x, y, z ; x_{a}, y_{a}, z_{a} ; E_{\lambda h}\right) \times \\
& \times V_{\delta}\left(x, y, z ; x_{a}, y_{a}, z_{a}\right) \Psi_{\lambda h}\left(x_{1}, y_{1}, z_{1}, x_{a}, y_{a}, z_{a}\right) .
\end{aligned}
$$

Substituting (17) into (20), we obtain:

$$
\Psi_{h}\left(x, y, z ; x_{a}, y_{a}, z_{a}\right)=\gamma G\left(x, y, z ; x_{a}, y_{a}, z_{a} ; E_{\lambda h}\right)\left(T \Psi_{h}\right)\left(x, y, z ; x_{a}, y_{a}, z_{a}\right),
$$

where:

$$
\left(T \Psi_{\lambda h}\right)\left(x, y, z ; x_{a}, y_{a}, z_{a}\right) \equiv \lim _{\substack{r \rightarrow r_{a} \\ \varphi \rightarrow \varphi_{a} \\ \theta \rightarrow \theta_{a}}}\left[1+\left(r-r_{a}\right) \frac{\partial}{\partial r}\right] \Psi_{\lambda h}\left(x, y, z ; x_{a}, y_{a}, z_{a}\right) .
$$

Acting by the operator $T$ on both sides of relation (21), we obtain an equation that determines dependence of the energy $E_{\lambda h}$ of the bound state of the $A^{+}$-center on the parameters of the QD, on the position of the impurity $R_{a}=\left(x_{a}, y_{a}, z_{a}\right)$, and on the temperature $T$ :

$$
\alpha=\frac{2 \pi \hbar^{2}}{m_{h}^{*}}(T G)\left(x_{a}, y_{a}, z_{a} ; x_{a}, y_{a}, z_{a} ; E_{\lambda h}\right),
$$

here, $\alpha$ is determined by the energy $E_{i}$ of the bound state of the same $A^{+}$-center in a massive semiconductor.

Then, for Green's function (29), taking into account (23) and (24), we obtain in the Bohr units:

$$
\begin{gathered}
G\left(x, y, z, x_{a}, y_{a}, z_{a} ; \eta_{\lambda h}^{2}\right)=-\frac{1}{\pi^{3 / 2} a_{n}^{2} E_{h}} \exp \left(-\frac{\left(x^{*}-x_{0}^{*}\right)^{2}+y^{* 2}+z^{* 2}+\left(x_{a}^{*}-x_{0}^{*}\right)^{2}+y_{a}^{* 2}+z_{a}^{* 2}}{2}\right) \times \\
\times \sum_{n_{1}, n_{2}, n_{3}} \frac{H_{n_{1}}\left(x^{*}-x_{0}^{*}\right) H_{n_{1}}\left(x_{a}^{*}-x_{0}^{*}\right)}{2^{n_{1} ! n_{1} !}} \frac{H_{n_{2}}\left(y^{*}\right) H_{n_{2}}\left(y_{a}^{*}\right)}{2^{n_{2} ! n_{2} !}} \frac{H_{n_{3}}\left(z^{*}\right) H_{n_{3}}\left(z_{a}^{*}\right)}{2^{n_{3} ! n_{3} !}} \times \\
\left\{-\eta_{h}^{2}-\beta_{h}^{*}-\frac{x_{0}^{* 2}}{4 \beta^{-2}}+i 4 \Gamma_{0}^{*}+\beta^{-1}\left(n_{1}+n_{2}+n_{3}+\frac{3}{2}\right)+\frac{k T}{E_{h}} \ln \left[4 \sinh \left(\frac{\Omega}{v_{L A} \sqrt{k T}}\right) \sinh \left(\frac{2 \Omega}{v_{T A} \sqrt{k T}}\right)\right]\right\}^{-1},
\end{gathered}
$$


where the next notations are introduced: $\eta_{h}^{2}=E_{h}^{Q D} / E_{h} ; R_{0}^{*}=R_{0} / a_{h} ; \beta=E_{h} / \hbar \omega_{n} ; a_{n}^{*}=a_{n} / a_{h}$; $\beta_{h}^{*}=e^{2} \beta_{h} / \varepsilon R_{0}^{*} E_{h} a_{h} ; \Gamma_{0}^{*}=\hbar \Gamma_{0} / 4 E_{h}$.

Further, given that:

$$
\begin{gathered}
\left(-\eta_{h}^{2}-\beta_{h}^{*}-\frac{x_{0}^{* 2} \beta^{-2}}{4 a_{h}^{* 2}}+i 4 \Gamma_{0}^{*}+\frac{k T}{E_{h}} \ln \left[4 \sinh \left(\frac{\Omega}{v_{L A} \sqrt{k T}}\right) \sinh \left(\frac{2 \Omega}{v_{T A} \sqrt{k T}}\right)\right]+n_{1}+n_{2}+n_{3}+\frac{3}{2}\right)^{-1}= \\
\beta^{-1} \int_{0}^{\infty} d t \exp \left[-t\left(-\beta\left(\eta_{h}^{2}+\beta_{h}^{*}+\frac{x_{0}^{* 2} \beta^{-2}}{4 a_{h}^{* 2}}-i 4 \Gamma_{0}^{*}\right)+\right.\right. \\
\left.+n_{1}+n_{2}+n_{3}+\frac{3}{2}+\frac{k T \beta}{E_{h}} \ln \left[4 \sinh \left(\frac{\Omega}{v_{L A} \sqrt{k T}}\right) \sinh \left(\frac{2 \Omega}{v_{T A} \sqrt{k T}}\right)\right]\right], \quad(25)
\end{gathered}
$$

the expression for the Green's function takes the following form:

$$
\begin{gathered}
G\left(x, y, z, x_{a}, y_{a}, z_{a}, \eta_{h}^{2}\right)=-\frac{1}{\pi^{\frac{3}{2}} a_{n}^{2} \beta E_{h}} \exp \left(-\frac{\left(x^{*}-x_{0}^{*}\right)^{2}+\left(x_{a}^{*}-x_{0}^{*}\right)^{2}+y^{2}+y_{a}^{2}+z^{2}+z_{a}^{2}}{2 a_{n}}\right) \times \\
\times \int_{0}^{\infty} d t \exp \left[-t\left(-\beta\left(\eta_{h}^{2}+\beta_{h}^{*}+\frac{x_{0}^{* 2} \beta^{-2}}{4 a_{h}^{* 2}}-i 4 \Gamma_{0}^{*}\right)+\frac{k T \beta}{E_{h}} \ln \left[4 \sinh \left(\frac{\Omega}{v_{L A} \sqrt{k T}}\right) \sinh \left(\frac{2 \Omega}{v_{T A} \sqrt{k T}}\right)\right]+\frac{3}{2}\right)\right] \times \\
\times \sum_{n_{1}=0}^{\infty}\left(\frac{e^{-t}}{2}\right)^{n_{1}} \frac{H_{n_{1}}\left(\frac{x-x_{0}}{a_{n}}\right) H_{n_{1}}\left(\frac{x_{a}-x_{0}}{a_{n}}\right)}{n_{1} !} \sum_{n_{2}=0}^{\infty}\left(\frac{e^{-t}}{2}\right)^{n_{2}} \frac{H_{n_{2}}\left(\frac{y}{a_{n}}\right) H_{n_{2}}\left(\frac{y_{a}}{a_{n}}\right)}{n_{2} !} \times \\
\times \sum_{n_{3}=0}^{\infty}\left(\frac{e^{-t}}{2}\right)^{n_{3}} \frac{H_{n_{3}}\left(\frac{z}{a_{n}}\right) H_{n_{3}}\left(\frac{z_{a}}{a_{n}}\right)}{n_{3} !} .
\end{gathered}
$$

Making summation over the quantum numbers $n_{1}, n_{2}, n_{3}$, and separating the divergent part of the expression for the Green's function (26), we obtain:

$$
\begin{aligned}
& G\left(r, R_{a}, \eta_{h}^{2}\right)=-\frac{1}{\pi^{\frac{3}{2}} a_{n}^{2} \beta E_{h}} \int_{0}^{\infty} d t \exp \left[-t\left(-\beta\left(\eta_{h}^{2}+\beta_{h}^{*}+\frac{x_{0}^{* 2} \beta^{-2}}{4 a_{h}^{* 2}}-i 4 \Gamma_{0}^{*}\right)+\frac{3}{2}+\right.\right. \\
& \left.\left.+\frac{k T \beta}{E_{h}} \ln \left[4 \sinh \left(\frac{\Omega}{v_{L A} \sqrt{k T}}\right) \sinh \left(\frac{2 \Omega}{v_{T A} \sqrt{k T}}\right)\right]\right)\right] \times \\
& \times\left\{(1-\exp (-t))^{-\frac{3}{2}} \exp \left(-\frac{r^{2}+R_{a}^{2}}{2 a_{n}}\right) \exp \left(\frac{2\left(r R_{a}\right) e^{-t}-\left(r^{2}+R_{a}^{2}\right) e^{-2 t}}{a_{n}\left(1-e^{-2 t}\right)}\right)-\frac{1}{t \sqrt{t}} \exp \left[-\frac{\left(r-R_{a}\right)^{2}}{2 a_{n}^{2} t}\right]\right\}- \\
& -\frac{\exp \left(-\sqrt{2\left(-\eta_{h}^{2}-\beta_{h}^{*}-\frac{x_{0}^{* 2} \beta^{-2}}{4 a_{h}^{* 2}}+i 4 \Gamma_{0}^{*}+\frac{k T}{E_{h}} \ln \left[4 \sinh \left(\frac{\Omega}{v_{L A} \sqrt{k T}}\right) \sinh \left(\frac{2 \Omega}{v_{T A} \sqrt{k T}}\right)\right]+\frac{3}{2}\right)} \frac{\left|r-R_{a}\right|}{a_{n}}\right)}{2 \pi^{\frac{3}{2}} a_{n} \beta E_{h}\left|r-R_{a}\right|} .
\end{aligned}
$$

Substituting (27) into (23) we obtain the dispersion equation that determines the dependence of the binding energy of a hole $E_{h}^{Q D}$ in the complex $A^{+}+e$ on the parameters of the QD, on temperature $T$, and on the electron quantum number $n$. Using the procedure of the zero-range potential method (see, for example, [3]) in combination with the one-instanton approximation [5], we obtain the dispersion equation that determines dependence of the hole binding energy in the complex $A^{+}+e$ on the temperature $T$ and on parameters of dissipative tunneling:

$$
\begin{aligned}
\eta_{i}=\sqrt{-\eta_{h}^{2}-\beta_{h}^{*}-\frac{x_{0}^{* 2} \beta^{-2}}{4 a_{h}^{* 2}}+i 4 \Gamma_{0}^{*}+\frac{k T}{E_{h}} \ln \left[4 \sinh \left(\frac{\Omega}{v_{L A} \sqrt{k T}}\right) \sinh \left(\frac{2 \Omega}{v_{T A} \sqrt{k T}}\right)\right]+\frac{3}{2}}+ \\
+\sqrt{\frac{2}{\beta \pi}} \int_{0}^{\infty} d t \exp \left\{-t \beta\left(-\eta_{h}^{2}-\beta_{h}^{*}-\frac{x_{0}^{* 2} \beta^{-2}}{4 a_{h}^{* 2}}+i 4 \Gamma_{0}^{*}+\frac{3}{2 \beta}+\frac{k T}{E_{h}} \times\right.\right. \\
\left.\left.\times \ln \left[4 \sinh \left(\frac{\Omega}{v_{L A} \sqrt{k T}}\right) \sinh \left(\frac{2 \Omega}{v_{T A} \sqrt{k T}}\right)\right]\right)\right\}\left[\frac{1}{2 t \sqrt{2 t}}-\left(1-e^{-2 t}\right)^{-\frac{3}{2}} \exp \left(-\frac{R_{a}^{*^{2}} \beta^{-1}}{2} \times \frac{1-e^{-t}}{1+e^{-t}}\right)\right],
\end{aligned}
$$


where $R_{a}^{*}=R_{a} / a_{n} ; \eta_{i}=\sqrt{\left|E_{i}\right| / E_{h}}, \eta_{h}=\sqrt{E_{h}^{(Q D)} / E_{h}}$.

It should be noted that the binding energy of a hole in the case under consideration is a complex quantity. Its real part determines the average binding energy of the resonant state of the $A^{+}$-center $\overline{E_{h}^{(Q D)}}=\operatorname{Re} E_{h}^{(Q D)}$, and the doubled imaginary part determines the broadening of the corresponding energy level $\Delta E_{h}=2 \operatorname{Im} E_{h}^{(Q D)}$. Fig. 1 shows the result of a numerical analysis of the dispersion equation for the case of a centered $A^{+}$-center $\left(R_{a}^{*}=0\right)$ at different values of the QD radius $R_{0}^{*}$. It was taken into account that the binding energy of the $A^{+}$-state is measured from the level of the ground state of the adiabatic oscillatory well.

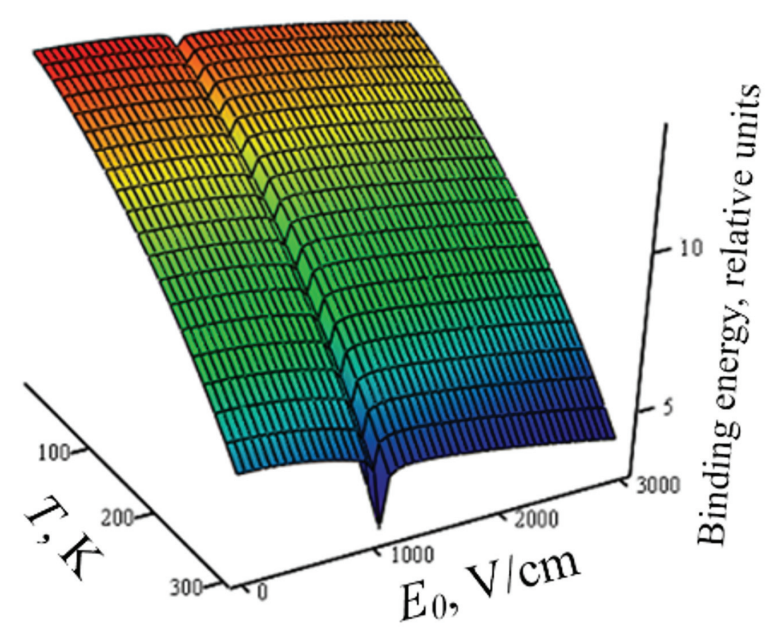

FIG. 1. Dependence of the binding energy of quasi-stationary states of a hole in the complex $A^{+}+e$ on the temperature $T$ and the magnitude of the external electric field $E_{0}$, for the following values of the QD parameters and dissipative tunneling $R_{0}^{*}=1\left(R_{0}^{*}=R_{0} / a_{h}\right.$ - QD radius $R_{0}$ in units of the effective Bohr radius of the hole $\left.a_{h}\right) ; U_{0}^{*}=350\left(U_{0}^{*}=U_{0} / E_{h}\right.$ is the amplitude of the adiabatic potential $U_{0}$ of an electron in units of the effective Bohr energy of a hole $\left.E_{h}\right), \eta_{i}=3$.

As can be seen from Fig. 1, that in the field dependence of the binding energy of the $A^{+}$-state, there are "dips" at a certain temperature. This is due to the effect of "tuning" the starting energy level of the $A^{+}$-state to the states caused by the hole-phonon interaction in the matrix surrounding the quantum dot, i.e. with the effect of resonant tunneling. The depth of the dip increases with increasing temperature, which is due to the dynamics of the temperature-dependent peak in the field dependence of the probability of dissipative tunneling [5]. A decrease in the binding energy of the $A^{+}$-state with an increase in the external electric field strength is associated with the Stark shift in energy and with the polarization of the $A^{+}$-center, and with an increase in temperature - with the broadening of energy levels and the corresponding "spreading" of the wave function of the $A^{+}$-state.

\section{Temperature and field dependences of the spectral intensity of recombination radiation in a quasi-zero-dimensional structure with impurity complexes}

Let us consider the process of radiative transition of an excited electron to the level of $A^{+}$-center. The Coulomb interaction of an electron with a hole is accompanied by a radiative transition of an electron to the energy level of $A^{+}$-center under conditions of tunneling decay of a quasi-stationary $A^{+}$-state. The energy spectrum of an electron in the size-quantized band can be represented as [6,7]:

$$
E_{n, l}=\frac{\tilde{X}_{n, l}^{2} E_{h}}{R_{0}^{* 2}}+k T \ln \left[4 \sinh \left(\frac{\Omega}{v_{L A} \sqrt{k T}}\right) \sinh \left(\frac{2 \Omega}{v_{T A} \sqrt{k T}}\right)\right],
$$

here $\tilde{X}_{n, l}$ is the root of the Bessel function of half-integer order $l+1 / 2$.

The wave function of an electron is given by expression (2).

The SIRR, taking into account the dispersion of QD sizes and the finite lifetime of the resonant $A^{+}$-state, is determined by an expression of the next form [8]:

$$
\Phi(\omega)=\frac{4 \omega^{2} \sqrt{\varepsilon} e^{2}}{c^{3} V}\left|\frac{P_{e h} e_{0}}{m_{0}}\right| \int \sum_{n l m}|M|^{2} P(u) \frac{\Gamma_{0}}{\frac{\hbar^{2} \Gamma_{0}^{2}}{4}+\left(E_{n l m}-E_{\lambda h}-\hbar \omega\right)^{2}} d u
$$


where $m_{0}$ - the free electron mass; $P_{e h}$ is the matrix element of the momentum operator at the Bloch amplitudes of band carriers; $\omega$ is the frequency of the emitted electromagnetic wave with polarization $e_{0} ; V$ is the QD volume; $P(u)$ - the Lifshitz-Slezov function [9]:

$$
P(u)= \begin{cases}\frac{3^{4} e u^{2} \exp [-1 /(1-2 u / 3)]}{2^{5 / 3}(u+3)^{7 / 3}(3 / 2-u)^{11 / 3}}, & u<\frac{3}{2}, \\ 0, & u>\frac{3}{2} .\end{cases}
$$

The wave function of $A^{+}$-state, in the case of a central location of the $A^{+}$center in the QD, has the form:

$$
\begin{array}{r}
\Psi_{h}(r)=-C \int_{0}^{\infty} d t \exp \left\{-\beta t\left(-\eta_{h}^{2}-\beta_{h}^{*}-\frac{x_{0}^{* 2}}{a_{n}^{* 4}}+i 4 \Gamma_{0}^{*}+\frac{k T}{E_{h} \beta} \ln \left[4 \sinh \left(\frac{\Omega}{v_{L A} \sqrt{k T}}\right) \sinh \left(\frac{2 \Omega}{v_{T A} \sqrt{k T}}\right)\right]+\right.\right. \\
\left.\left.+\frac{3}{2 \beta}\right)\right\} \times(1-\exp (-t))^{-3 / 2} \exp \left(-\frac{\left(1+e^{-2 t}\right)}{\left(1-e^{-2 t}\right)} \times \frac{r^{2}}{2 a_{n}^{2}}\right),
\end{array}
$$

where $C$ is the normalization factor, determined by an expression of the next form:

$$
C=\left\{\frac{2 \sqrt{\pi}}{\left[\eta_{h}^{2}(T)\right]^{2}} \frac{\Gamma\left(1+\frac{\eta_{h}^{2}(T)}{2}\right) \cdot a_{n}^{3}}{\Gamma\left(\frac{\eta_{h}^{2}(T)-1}{2}\right)}\left[\frac{\eta_{h}^{2}(T)}{2}\left(\Psi\left(\frac{\eta_{h}^{2}(T)}{2}+1\right)-\Psi\left(\frac{\eta_{h}^{2}(T)}{2}-\frac{1}{2}\right)-1\right)\right]\right\}^{-\frac{1}{2}} .
$$

Here, $\eta_{\lambda h}^{2}(T)$ is determined by the dispersion equation (28).

The matrix element of the radiative transition of an excited electron to the level of $A^{+}$-center in the dipole approximation has the form:

$$
M=i \lambda_{0} \sqrt{\frac{2 \pi \alpha^{*} I_{0}}{\omega}}\left(E_{n, l, m}-E_{h}\right)\left\langle\Psi_{h}(r)\left|\left(\vec{e}_{\lambda}, \vec{r}\right)\right| \Psi_{n, l, m}(\rho, \theta, \varphi)\right\rangle .
$$

Taking into account (2), (28) and (32), the matrix element of the radiative recombination transition of an electron from the ground state of the size-quantized band to the level of $A^{+}$-center in the QD can be represented as:

$$
\begin{aligned}
& M=\frac{a_{h}^{-1} 2^{-\frac{3}{2}} \pi^{-\frac{5}{4}} \sqrt{3} \beta^{\frac{1}{2}}}{\sqrt{2 \pi} R_{0}^{* 4} J_{\frac{3}{2}}\left(\tilde{X}_{n, 1}\right)}\left[\frac{\Gamma\left(1+\frac{\eta_{h}^{2}(T)}{2}\right)}{\left[\eta_{h}^{2}(T)\right]^{2} \Gamma\left(\frac{\eta_{h}^{2}(T)-1}{2}\right)} \times\right. \\
& \left.\times\left[\frac{\eta_{h}^{2}(T)}{2}\left(\Psi\left(\frac{\eta_{h}^{2}(T)}{2}+1\right)-\Psi\left(\frac{\eta_{h}^{2}(T)}{2}-\frac{1}{2}\right)-1\right)\right]\right]^{-\frac{1}{2}} \int_{0}^{+\infty} \int_{0}^{\pi} \int_{0}^{2 \pi} r^{* 2} d r^{*} \cos \theta \sin \theta d \theta d \varphi \times \\
& \times \int_{0}^{\infty} d t \exp \left[-t\left(-\eta_{h}^{2} \beta-\beta \beta_{h}^{*}-\frac{x_{0}^{* 2} \beta}{a_{n}^{* 4}}+i 4 \beta \Gamma_{0}^{*}+\frac{k T \beta}{E_{h}} \ln \left[4 \sinh \left(\frac{\Omega_{n}}{v_{L A} \sqrt{k T}}\right) \sinh \left(\frac{2 \Omega_{n}}{v_{T A} \sqrt{k T}}\right)\right]+\frac{3}{2}\right)\right] \times \\
& \times(1-\exp (-t))^{-\frac{3}{2}} \exp \left(-\frac{\left(1+e^{-2 t}\right)}{\left(1-e^{-2 t}\right)} \times \frac{r^{* 2}}{2}\right) Y_{l, m}(\theta, \varphi) \frac{J_{l+\frac{3}{2}}\left(\frac{\tilde{X}_{n, l}}{R_{0}^{*}} r^{*}\right)}{\sqrt{r^{*}} J_{l+\frac{3}{2}}\left(\tilde{X}_{n, l}\right)},
\end{aligned}
$$

where $R_{0}^{*}=R_{0} / a_{h}$.

Calculation of (35) leads to integrals giving selection rules for quantum numbers $m$ and $l$ :

$$
\begin{gathered}
\int_{0}^{2 \pi} \exp (i m \varphi) d \varphi= \begin{cases}2 \pi, & \text { if } m=0, \\
0, & \text { if } m \neq 0,\end{cases} \\
\int_{0}^{\pi} P_{l}(\cos \theta) \cos \theta \sin \theta d \theta= \begin{cases}\frac{2}{3}, & \text { if } l=1, \\
0, & \text { if } l \neq 1 .\end{cases}
\end{gathered}
$$

Thus, the radiative transition of an electron to the level of the $A^{+}$-center is possible only from states with the values of quantum numbers $l=0$ and $m=0$. 
The remaining integral over the radial coordinate $r^{*}$ has the form:

$$
\begin{aligned}
\int_{0}^{\infty} d r^{*} r^{* 3 / 2} J_{l+\frac{3}{2}}\left(\frac{\tilde{X}_{n, l} r^{*}}{R_{0}^{*}}\right) \exp \left(-\frac{\left(1+e^{-2 t}\right)}{2\left(1-e^{-2 t}\right)} r^{* 2}\right)= \\
\sqrt{\frac{\tilde{X}_{n, l}}{R_{0}^{*}}} \exp \left(-\frac{1}{2}\left(\frac{1-e^{-2 t}}{1+e^{-2 t}}\right)\left(\frac{\tilde{X}_{n, l}}{R_{0}^{*}}\right)^{2}\right)\left(\frac{1-e^{-2 t}}{1+e^{-2 t}}\right)^{3 / 2} .
\end{aligned}
$$

Taking into account (36), (37), and (38), for the square of the matrix element (35), we have:

$$
\begin{array}{r}
|M|^{2}=\frac{2^{-2} \pi^{-5 / 2} \beta_{h} \tilde{X}_{n, 1}}{a_{h}^{2} R_{0}^{* 9}\left|J_{\frac{3}{2}}\left(\tilde{X}_{n, 1}\right) J_{\frac{5}{2}}\left(\tilde{X}_{n, 1}\right)\right|^{2}}\left|\frac{\Gamma\left(1+\frac{\eta_{h}^{2}}{2}\right)}{\left[\eta_{h}^{2}\right]^{2} \Gamma\left(\frac{\eta_{h}^{2}-1}{2}\right)}\left[\frac{\eta_{h}^{2}}{2}\left(\Psi\left(\frac{\eta_{h}^{2}}{2}+1\right)-\Psi\left(\frac{\eta_{h}^{2}}{2}-\frac{1}{2}\right)-1\right)\right]\right|^{-1} \times \\
\times \mid \int_{0}^{\infty} d t \exp \left[-t\left(-\eta_{h}^{2} \beta-\beta \beta_{h}^{*}-\frac{x_{0}^{* 2} \beta}{a_{n}^{* 4}}+i 4 \beta \Gamma_{0}^{*}+\frac{k T \beta}{E_{h}} \ln \left[4 \sinh \left(\frac{\Omega}{v_{L A} \sqrt{k T}}\right) \sinh \left(\frac{2 \Omega}{v_{T A} \sqrt{k T}}\right)\right]+\frac{3}{2}\right)\right] \times \\
\times\left.(1-\exp (-t))^{-3 / 2} \exp \left(-\frac{1}{2}\left(\frac{1-e^{-2 t}}{1+e^{-2 t}}\right)\left(\frac{\tilde{X}_{n, 1}}{R_{0}^{*}}\right)^{2}\right)\left(\frac{1-e^{-2 t}}{1+e^{-2 t}}\right)^{3 / 2}\right|^{2}
\end{array}
$$

Taking into account (31) and (39) for the spectral intensity of recombination radiation (SIRR) in QD (30), we can write:

$$
\begin{gathered}
\Phi(X, T)=\Phi_{0} \times \frac{a_{h}^{4} \beta_{h} \tilde{X}_{n, 1} X^{2}}{R_{0}^{* 12}\left|J_{\frac{3}{2}}\left(\tilde{X}_{n, 1}\right) J_{\frac{5}{2}}\left(\tilde{X}_{n, 1}\right)\right|^{2}} \times \int_{0}^{3 / 2} d u P(u) \times \\
\times \mid \frac{\Gamma\left(1+\frac{\eta_{h}^{2}}{2}\right)}{\left(\eta_{h}^{2}\right)^{2} \Gamma\left(\frac{\eta_{h}^{2}-1}{2}\right)}\left[\left.\frac{\eta_{h}^{2}}{2}\left(\Psi\left(\frac{\eta_{h}^{2}}{2}+1\right)-\Psi\left(\frac{\eta_{h}^{2}}{2}-\frac{1}{2}\right)-1\right)\right|^{-1} \times\right. \\
\times \int_{0}^{\infty} d t \exp \left[-t\left(-\eta_{h}^{2} \beta-\beta \beta_{h}^{*}-\frac{x_{0}^{* 2} \beta}{a_{n}^{* 4}}+i 4 \beta \Gamma_{0}^{*}+\frac{k T \beta}{E_{h}} \ln \left[4 \sinh \left(\frac{\Omega}{v_{L A} \sqrt{k T}}\right) \sinh \left(\frac{2 \Omega}{v_{T A} \sqrt{k T}}\right)\right]+\frac{3}{2}\right)\right] \times \\
\times\left.(1-\exp (-t))^{-3 / 2} \exp \left(-\frac{1}{2}\left(\frac{1-e^{-2 t}}{1+e^{-2 t}}\right)\left(\frac{\tilde{X}_{n, 1}}{R_{0}^{*}}\right)^{2}\right)\left(\frac{1-e^{-2 t}}{1+e^{-2 t}}\right)^{3 / 2}\right|^{2} \times \\
\times \frac{\Gamma_{0}^{*}}{\Gamma_{0}^{* 2}+\left(\frac{X_{n, 1}^{2}}{R_{0}^{* 2}}+\frac{k T}{E_{h}} \ln \left[4 \sinh \left(\frac{\Omega}{v_{L A} \sqrt{k T}}\right) \sinh \left(\frac{2 \Omega}{v_{T A} \sqrt{k T}}\right)\right]-\eta_{\lambda h}^{2}-X\right)^{2}}, \quad(40)
\end{gathered}
$$

where $X=\hbar \omega / E_{h} ; \Phi_{0}=\sqrt{\varepsilon} e^{2}\left|P_{e h} e_{0}\right| / 4 \pi^{\frac{5}{2}} \hbar^{3} c^{3} m_{0}$.

Figure 2 shows the SIRR dependence on the magnitude of the external electric field $E_{0}$. It can be seen that the decrease in the SIRR value with increasing of $E_{0}$ is accompanied by "dips", that appear at certain values of the external electric field strength and temperature. In [5], it is shown that variation of the strength of the external electric field can lead to transformation of the shape of the double-well oscillatory potential, which simulates the system "QD - surrounding matrix", while the transition to the symmetric shape of the double-well oscillatory potential is accompanied by the appearance of a peak in the field dependence of tunneling probability. Thus, the nature of the dip appears to be related to the effect of resonant tunneling, when the double-well oscillator potential becomes symmetric. An increase in the SIRR value with the temperature increasing (Fig. 2) is associated with an increase in the overlap integral of the wave functions of the initial and final states due to temperature smearing of energy levels. It should be noted that the presence of dissipative tunneling makes the optics of quantum dots very sensitive to the parameters of the surrounding matrix, which determine, respectively, the frequency of the phonon mode $\epsilon_{L}$ and the constant of interaction with the contact medium (with the heat-bath) $\epsilon_{C}$. With an increase in the value of $\epsilon_{L}$, the wave function of the $A^{+}$-state "spreads" due to the hole-phonon interaction, which is accompanied by a decrease in the SIRR value. An increase in the parameter $\epsilon_{C}$ leads to an increase in the "viscosity" of the surrounding matrix, i.e. to a decrease 


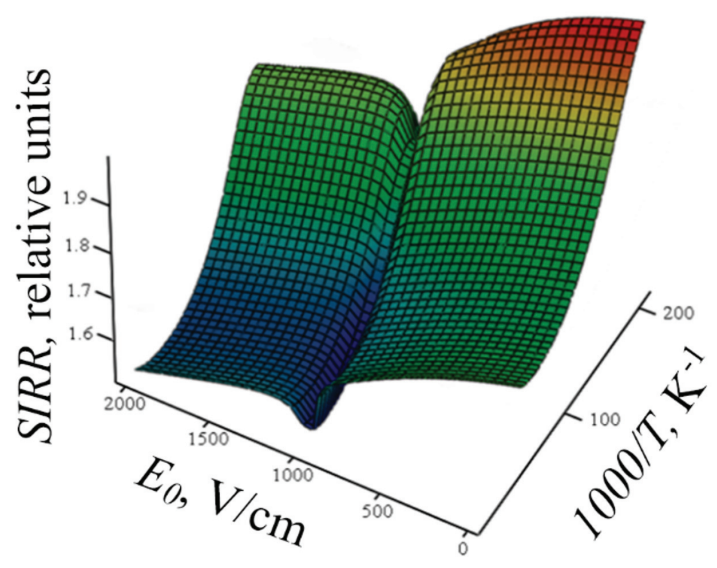

FIG. 2. The SIRR dependence on the inverse temperature $1000 / T$ and the external electric field strength $E_{0}$ at $R_{0}^{*}=1 ; U_{0}^{*}=350 ; \eta_{i}=3$.

in the probability of dissipative tunneling. As a result, the binding energy of the $A^{+}$-state increases, and the overlap integral of the wave functions of the initial and final states decreases, which leads to a decrease in the SIRR value.

\section{Conclusion}

The possibility of the existence of a luminescence temperature quenching channel in semiconductor quasi-zerodimensional structures with " $A^{+}+e$ " impurity complexes associated with dissipative tunneling of a hole into the matrix, surrounding the QD, has been theoretically substantiated. It is shown that this channel "triggers" efficiently at the temperature and strength of the external electric field, for which the double-well oscillatory potential, simulating the "quantum dot - surrounding matrix" system, becomes symmetric (resonant tunneling effect).

\section{Acknowledgements}

The present study was supported by the Ministry of Education and Science of the Russian Federation (Project No. 0748-2020-0012).

\section{References}

[1] Shamirzaev T.S., Klochikhin A.A, et. al. Photoluminescence of germanium quantum dots grown in silicon on a $\mathrm{SiO}_{2}$ monolayer. Physics of the Solid State, 2005, 47 (1), P. 82-85.

[2] Reznitsky A.N., Seksenbaev K.S, Permogorov S.A. Temperature dependence of the photoluminescence intensity of self-organized CdTe quantum dots in a ZnTe matrix under different excitation conditions. Physics of the Solid State, 2012, 54 (1), P. 123-133.

[3] Krevchik V.D., Levashov A.V. Energy spectrum of the complex $A^{+}+e$ in a quantum dot in the adiabatic approximation. Physics of the Solid State, 2006, 48 (3), P. 589-592.

[4] Kusmartsev F.V., Krevchik V.D, et al. Phonon assisted resonant tunnelling and its phonons control. JETP Letters, 2016, 104, P. $392-397$.

[5] Leggett A.J., Krevchik V.D, et al. Controllable dissipative tunneling. Tunnel transport in low-dimensional systems. Fizmatlit, Moscow, 20112012. 496 p.

[6] Vainshtein I.A., Zatsepin A.F, Kortov V.S. Applicability of the empirical Varshni relation for the temperature dependence of the width of the band gap. Physics of the Solid State, 1999, 41 (6), P. 905-908.

[7] Ridley B. Quantum processes in semiconductors. Mir, Moscow, 1986.

[8] Zhou Hai-Yang, Gu Shi-Wei, Shi Yao-Ming. Electronic and shallow impurity states in semiconductor heterostructures under an applied electric field Commun. Theor. Phys., 2005, 44, P. 375-380.

[9] Lifshits I.M., Slezov V.V. On the kinetics of diffusion decomposition of supersaturated solid solutions. JETP: Journal of Experimental and Theoretical Physics (ZhETF), 1958, 35 (2), P. 479-492. 